\title{
A rare case of heterotopic pregnancy: an avalanche of catastrophe
}

\author{
Rangan Bhattacharya*, Rohini Raut, Beena Kumari, D. C. Hojai
}

Department of Obstetrics and Gynecology, Dr. B. R. Ambedkar Memorial Central Railway Hospital, Byculla, Mumbai, India

Received: 02 September 2020

Accepted: 08 October 2020

\section{*Correspondence:}

Dr. Rangan Bhattacharya,

E-mail: rbhattac@ rediffmail.com

Copyright: ( ) the author(s), publisher and licensee Medip Academy. This is an open-access article distributed under the terms of the Creative Commons Attribution Non-Commercial License, which permits unrestricted non-commercial use, distribution, and reproduction in any medium, provided the original work is properly cited.

\begin{abstract}
An ectopic pregnancy is the development of an embryo outside the uterus, a major cause of maternal morbidity and mortality. Heterotopic pregnancy is when an intrauterine implanted embryo develops simultaneously along with an extra uterine implantation. With rising incidence of assisted reproductive technology (ART), incidence of ectopic pregnancy is on a rise. We report a case of an elderly patient, conceived by in vitro fertilization and embryo transfer (IVF-ET), who had a catastrophe of events following conception, where she had an intrauterine twin gestation and an ectopic gestation in the left fallopian tube. She presented with vomiting and on ultrasound scan, we found a twin intra uterine gestation, with demise of one twin with no sign of ectopic implantation. Initially we decided to continue pregnancy. However, she had persistent spotting per vagina, and on a repeat ultrasound scan there was intrauterine demise of the other foetus also. Her uterus was then evacuated under anaesthesia. Post operatively, she became hemodynamic ally unstable and ultrasound was inconclusive of the cause. So, we subjected her to an MRI which showed a left adnexal ruptured ectopic pregnancy with hemoperitoneum. She was immediately taken up for exploratory laparotomy and left salpingectomy was done hemoperitoneum evacuated. Due to timely intervention and use of advanced techniques for diagnosis, she recovered successfully. Thus, heterotopic pregnancy can be a rare, life threatening complication of IVF, a vigilant eye and not shying away from the use of novel and advanced techniques for diagnosis, can help in early diagnosis and timely intervention and thus prove to be lifesaving.
\end{abstract}

Keywords: Ectopic pregnancy, Heterotopic pregnancy, MRI in pregnancy, In vitro fertilization and embryo transfer, Assisted reproductive technology, Multifoetal gestation

\section{INTRODUCTION}

Ectopic pregnancy or the implantation of an embryo outside the uterus can be a life-threatening obstetric complication. Its incidence is about $1.3 \%$ to $2 \%$ of natural pregnancies and around $1.7 \%$ for pregnancies occurring with assisted reproductive technologies (ART). ${ }^{1}$

Most ectopic pregnancies $(90 \%)$ implant in the tubal ampulla, while $2.5 \%$ of cases are identified in the tubal interstitial portion. Only in approximately $2 \%$ of the cases EP occur in different regions: cornual, cervical.
A heterotopic pregnancy (HP) is the result of the increased use of ART. HP is a multiple pregnancy with different site of implantation of embryos: one intrauterine, while the other(s) outside. ${ }^{2}$ In fact HP is quite rare among the general population (1/7,963$1 / 30,000)$, while its incidence in ART pregnancies vary between $1 / 100$ to $1 / 3,600 .^{3,4}$

Though an ultrasound scan and serum beta HCG is good enough for the diagnosis, however, it can be misleading at times and may require good clinical skills and use of advanced imaging technology. 
Due to a missed diagnosis, the patient can present in obstetric shock and if not intervened timely, can lead to irreversible shock and multiple organ damage and sometimes death.

\section{CASE REPORT}

A 33-year-old patient, gravida 2 abortion 1 (G2A1), married for 10 years, conceived by in vitro fertilisation, reported for the first time at 8 weeks gestation to our outpatient department with history of two episodes of vomiting and dizziness since 2 days. Her previous pregnancy resulted in a missed abortion at 2 months gestation, 6 years back. This was a spontaneous conception. She was also a known hypothyroid, on medications.

On examination of this patient, she was vitally stable with no signs of dehydration. There were no complaints of spotting or bleeding per vagina. Considering this as a precious pregnancy, she was admitted at our hospital and was given antiemetics as and when required and was kept under observation. She was then subjected for her first obstetric ultrasound scan, which showed live dichorionic twins of both 8 weeks gestation, thus making the cause of her hyperemesis evident. She was kept under observation and was discharged with required medications after her symptoms subsided.

After about 2 weeks, the same patient came back to us with complaints to spotting per vaginum. On speculum examination, cervical os was closed, with minimal bleeding through os. She was admitted, kept under observation, given bed rest, started on oral and injectable progesterone's. She was then subjected for another ultrasound scan. This scan showed two G-sacs, one live foetus of 10 weeks and no cardiac activity in the second foetus.

As this was a pregnancy with ART, hence a precious pregnancy, the patient and her husband decided to continue with the pregnancy with one twin IUFD, after having explained to them the risks, the pros and cons of doing so. She was given appropriate pregnancy support and was regularly monitored for any signs of infection or abortion. She was kept in the hospital and about 5 days later, she complained of increased spotting per vagina. This time on speculum examination, she had considerable amount of bleeding with clots and the cervical os was partially open. She was immediately subjected for an ultrasound scan, which revealed, Intra uterine foetal demise of both twins with an open cervical os. She was then taken up for check curettage and the products of conception were removed. Post operatively, she was stable and all her blood examination was normal, Thyroid function under control and euglycemic.

On post-operative day 3, patient complained of constipation, pain in lower abdomen and urgency but inadequate voiding of urine. Considering it could be a postoperative complication, we treated her symptomatically, and showed her to surgeons. On examination, she appeared pale, was slightly tachycardic, had a guarded abdomen and generalised abdominal tenderness with sluggish bowel sounds. Her investigations showed a fall of haemoglobin from $9.2 \mathrm{~g} \%$ to $6 \mathrm{~g} \%$, abdominal X-ray showed multiple air-fluid levels and ultrasound scan revealed a mild subhepatic collection.

Unsure of whether this was plain paralytic ileus or hepatic abscess or intraoperative perforation of the uterus; she was subjected for an MRI after transfusing one pint of blood.

To our surprise, the MRI revealed a large pelvic hematoma extending into the pouch of douglas, up to the os, encircling the uterus and bilateral ovaries, with associated mixed signal intensity cystic hemorrhagic lesion in the left adnexa suggestive of ruptured left tubal ectopic pregnancy with pelvic haemorrhage. Possibility of uterine perforation is less likely.

Patient was immediately taken up for surgical exploration and was found to have a massive hemoperitoneum. A left total salpingectomy was done. Patient was transfused with 2 pints of whole blood intra operatively, and 2 pints postoperatively. Patient recovered uneventfully and was discharged on the 8th day, post operatively, after suture removal.

\section{DISCUSSION}

Ectopic pregnancy (EP), which is the implantation of a fertilized ovum outside the endometrial cavity and has an approximate incidence of 1.5-2.0\% in all pregnancies, is a potentially life-threatening disease. ${ }^{5}$ With increasing in vitro fertilization (IVF) procedures, there is an increase of ectopic pregnancies. ${ }^{6} \mathrm{EP}$ is the leading cause of maternal death during the first trimester of pregnancy, accounting for approximately $10 \%$ of all pregnancy-related deaths.

Our case is that of a 33 year old lady who presented with atypical symptoms of ectopic pregnancy. The risk of EP increases with advancing maternal age. ${ }^{7,8}$ Some researchers attributed it to some age-related factors, such as: Possible tubal scarring from PID major gonococcal and chlamydial epidemics and changes in tubal function leading to delay in ovum transport and tubal implantation. ${ }^{9-11}$ A 3- to 4-fold increase in the risk for developing an EP exists in the age group of 35-44 years as compared to women aged 15-24 years. The probable cause for this high incidence is the progressive loss of the myoelectrical activity in the fallopian tube with age, which is responsible for tubal motility. ${ }^{12}$

In our case, the lady had a history of a previous spontaneous abortion 5 years before her present pregnancy. The risk of ectopic pregnancy is higher in women with a history of spontaneous abortion. Several 
other studies showed the association of prior spontaneous abortion with increased risk of EP. With regard to the available evidence, the cause of this relationship is most likely due to infection, hormonal imbalance or immunologic factors and intra uterine adhesions. ${ }^{13}$

The lady, in our case had secondary infertility and was trying to conceive since 4 years after her first abortion. Infertility is a problem affecting $8-12 \%$ of couples worldwide. A strong association between history of subfertility and risk of EP was also detected which may be due to a significant role of hyperstimulation, with high estrogen levels. ${ }^{14,15}$

The patient, in our case was receiving ART and had undergone IVF-ET. The prevalence of EP following ART ranges between 2.1 to $8.6 \%$ of all pregnancies and it can reach up to $11 \%$ in female patients with a history of tubal factor infertility. In cases of EP post IVF-ET, in which passage of the embryo along the Fallopian tube does not occur, additional factors that prevent intrauterine implantation must precede ectopic implantation of the embryo. One study has compared tubal pathology in natural and IVF ectopic pregnancies, using E-cadherin as a marker of implantation potential. ${ }^{16,17}$

Another explanation for EP during IVF-ET would be impairment of tubal function and endometrial receptivity with ectopic implantation occurring following failure of the normal biological interactions between endometrium, Fallopian tube and embryo due to controlled ovarian stimulation (COS) and the subsequent alteration in hormonal milieu. ${ }^{18,19}$

We are reporting a case of a patient with multi fetal gestation following IVF-ET. Multifetal gestation is the most common side effect of ART. The rate of natural twinning is relatively stable worldwide at under $2 \% .{ }^{20} \mathrm{It}$ has been estimated that births resulting from infertility treatments account for about $1 \%$ to $3 \%$ of all single live births, $30 \%$ to $50 \%$ of twin births, and for more than $75 \%$ of higher order multiple births. ${ }^{20}$ The risk of multiple births associated with IVF is directly related to the number of embryos transferred in each cycle. The practice of transferring more than 1 embryo goes back to the early days of IVF when embryo quality and implantation rates were quite low and placing multiple embryos was performed to compensate for low implantation potential. Recent estimates of the average implantation rate are around $50 \%$, but this varies considerably according to the woman's age.

In our case, the patient was infertile, had undergone treatment for the same and had an early pregnancy loss, following IVF-ET. The risk of miscarriage in women with infertility has been reported to range widely from $7 \%$ to as high as $70 \% . .^{21-23}$ It has been reported that pregnancy following IVF treatment has a particularly high loss rate, with several reports on rates well over $30 \%$, similar to that observed in women with a history of recurrent miscarriage. A review of the literature has shown that pregnancy loss rate after documentation of foetal heart (foetal loss) was $3-6 \%$ in patients with no history of infertility and/or recurrent miscarriages. In the infertile populations this ranges from 7 to $15 \%$. $^{24-26}$

The patient was a case of heterotopic pregnancy. Recent data indicate that the rate of heterotopic pregnancy is high due to assisted reproduction and is approximately 1 in 7000 overall and as high as 1 in 900 with ovulation induction. ${ }^{27}$ The increased incidence of multiple pregnancies with ovulation induction and IVF increases the risk of both ectopic and heterotypic gestation. The hydrostatic forces generated during embryo transfer may also contribute to the increased risk. ${ }^{28}$

Majority of the reported heterotopic pregnancies are of singleton intrauterine pregnancies. Triplet and quadruplet heterotopic pregnancies have also been reported, though extremely rare. ${ }^{29,30}$ It can be multiple as well. They can be seen frequently with assisted conceptions.

Around $50 \%$ of heterotopic pregnancies are asymptomatic. ${ }^{31}$ When symptomatic, the main clinical manifestations are abdominal pain due to peritoneal irritation, adnexal mass with or without vaginal bleeding and hypovolemic shock, similar to our case.

Heterotopic pregnancy is often difficult to diagnose as intrauterine gestation with hemorrhagic corpus luteum can simulate heterotopic/ectopic gestation both clinically and on ultrasound. ${ }^{31}$ Other surgical conditions of acute abdomen can also simulate heterotopic gestation clinically and hence the difficulty in clinical diagnosis. Bicornuate uterus with gestation in both cavities may also simulate a heterotopic pregnancy.

Ultrasound scan may often be indeterminate in the diagnosis of a heterotopic pregnancy, and in such cases, MRI of the pelvis may be used to assist in the diagnosis, similar to our case. Structures located in the adnexal region that are similar to the gestational sac, or even cystic formations, may be identified on MRI. Occasionally, it is possible to observe a thickened tubal wall with cystic content within, or even the presence of hematomas due to the tubal rupture (low T2 signal intensity). ${ }^{32}$

Once the condition was diagnosed the patient underwent emergency laparotomy with salpingectomy. A massive hemoperitoenum was evacuated and the blood loss was replaced. This treatment was guided by a timely MRI. This is similar to a case report published in 2012 .

\section{CONCLUSION}

In conclusion, we presented a case of heterotopic pregnancy in which patient had shown atypical symptoms and a regular ultrasound scan was unable to diagnose, until the rupture of the tubal ectopic. Thus, an MRI was 
essential to complement the diagnosis, thereby allowing adequate and timely surgical treatment. Because of the rarity of heterotopic pregnancy, we recommend all IVF patients to be monitored carefully and keep a vigilant eye on the signs and symptoms of ectopic pregnancy, and make use of evolving techniques like MRI to confirm diagnosis, especially in situations where ultrasound scan is in conclusive, so that there can be timely intervention, thus saving the life of the patient.

\section{Funding: No funding sources}

Conflict of interest: None declared

Ethical approval: Not required

\section{REFERENCES}

1. Farquhar CM. Ectopic pregnancy. Lanc. 2005;366(9485):583-91.

2. Talbot K, Simpson R, Price N, Jackson SR Heterotopic pregnancy. J Obstet Gynaecol. 2011;31:7-12

3. Barrenetxea G, Barinaga-Rementeria L, Lopez de Larruzea A, Agirregoikoa JA, Mandiola M, et al. Heterotopic pregnancy: Two cases and a comparative review. Fertil Steril. 2007;87:417e9.

4. Felekis T, Akrivis C, Tsirkas P, Korkontzelos I. Heterotopic triplet pregnancy after in vitro fertilization with favorable outcome of the intrauterine twin pregnancy subsequent to surgical treatment of the tubal pregnancy. Case Rep Obstet Gynecol. 2014:356131.

5. Chang J, Elam-Evans LD, Berg CJ, Herndon J, Flowers L, Seed KA, et al. Pregnancy-related mortality surveillance-United States, 1991-1999. MMWR Surve Summ. 2003;52(2):1-8

6. Ramer I, Kanninen TT, Sisti G, Witkin SS, Spandorfer SD. The serum brain-derived neurotrophic factor concentration prior to initiation of an in vitro fertilization cycle predicts outcome. $\mathrm{J}$ Reprod Immunol. 2016;116:46-9.

7. Coste J, Job-Spira N, Fernandez H, Papiernik E, Spira A. Risk factors for ectopic pregnancy: A casecontrol study in France, with special focus on infectious factors. Am J Epidemiol. 1991;133:83949.

8. Mäkinen JI, Erkkola RU, Laippala PJ. Causes of the increase in the incidence of ectopic pregnancy. A study on 1017 patients from 1966 to 1985 in Turku, Finland. Am J Obstet Gynecol. 1989;160:642-6.

9. Egger M, Low N, Smith GD, Lindblom B, Herrmann B. Screening for chlamydial infections and the risk of ectopic pregnancy in a county in Sweden: Ecological analysis. Brit Med J. 1998;316:1776-80.

10. Kamwendo F, Forslin L, Bodin L, Danielsson D. Epidemiology of ectopic pregnancy during a 28 year period and the role of pelvic inflammatory disease. Sex Transm Infect. 2000;76:28-32.

11. Bouyer J, Coste J, Shojaei T, Pouly JL, Fernandez H, Gerbaud L, et al. Risk factors for ectopic pregnancy: A comprehensive analysis based on a large case- control, population-based study in France. Am J Epidemiol. 2003;157:185-94.

12. Patil M. Ectopic pregnancy after infertility treatment. J Hum Reprod Sci. 2012;5(2):154-65.

13. Honoré LH. A significant association between spontaneous abortion and tubal ectopic pregnancy. Fertil Steril. 1979;32:401-2.

14. Mascarenhas MN, Flaxman SR, Boerma T, Vanderpoel S, Stevens GA. National, regional, and global trends in infertility prevalence since 1990: a systematic analysis of 277 health surveys. PLoS Med. 2012;9.

15. Gemzell C, Guillome J, Wang CF. Ectopic pregnancy following treatment with human gonadotropins. Am J Obstet Gynecol. 1982;143:7615 .

16. Clayton HB, Schieve LA, Peterson HB, Jamieson DJ, Reynolds MA, Wright VC. Ectopic pregnancy risk with assisted reproductive technology procedures. Obstet Gynecol. 2006;107:595-604.

17. Revel A, Ophir I, Koler M, Achache H, Prus D. Changing etiology of tubal pregnancy following IVF. Hum Reprod. 2008;23:1372-6.

18. Shao R, Nutu M, Weijdegard B, Egecioglu E, Fernandez-Rodriguez J, Karlsson-Lindahl L, et al. Clomiphene citrate causes aberrant tubal apoptosis and estrogen receptor activation in rat fallopian tube: implications for tubal ectopic pregnancy. Biol Reprod. 2009;80:1262-71.

19. Jia-Rong Z, Shuang-Di L, Xiao-Ping W. Eutopic or ectopic pregnancy: a competition between signals derived from the endometrium and the fallopian tube for blastocyst implantation. Placen. 2009;30:835-9

20. Multiple gestation pregnancy. The ESHRE Capri Workshop Group. Hum Reprod. 2000;15(8):185664.

21. Fauser BC, Devroey P, Macklon NS. Multiple birth resulting from ovarian stimulation for subfertility treatment. Lancet. 2005;365(9473):1807-16.

22. Molo MW, Kelly M, Balos R, Mullaney K, Radwanska E. Incidence of fetal loss in infertility patients after detection of fetal heart activity with early transvaginal ultrasound. J Reproduct Medic. 1993;38(10):804-6.

23. Hakim RB, Gray RH, Zacur H. Infertility and early pregnancy loss. Americ J Obstet Gynecol. 1995;172(5):1510-7.

24. Liu H.-C., Rosenwaks Z. Early pregnancy wastage in IVF (in vitro fertilization) patients. J Vit Fertilizat Embr Transf. 1991;8(2):65-72.

25. Hill LM, Guzick D, Fries J, Hixson J. Fetal loss rate after ultrasonically documented cardiac activity between 6 and 14 weeks, menstrual age. J Clinic Ultrasou. 1991;19(4):221-3.

26. Laufer MR, Ecker JL, Hill JA. Pregnancy outcome following ultrasound-detected fetal cardiac activity in women with a history of multiple spontaneous abortions. J Soc Gynecol Investigat. 1994;1(2):13842 . 
27. Glassner MJ, Aron E, Eskin BA. Ovulation induction with clomiphene and the rise in heterotopic pregnancies: A report of two cases. J Reprod Med. 1990;35:175-8.

28. Lyons EA, Levi CS, Sidney M. In: Dashefsky in diagnostic ultrasound. 2nd ed. Rumak CM, Wilson SR, Charboneau WK, editors. 1998;(2):999.

29. Alsunaidi MI. An unexpected spontaneous triplet heterotopic pregnancy. Saudi Med J. 2005;26:136-8.

30. Sherer DM, Scibetta JJ, Sanko SR. Heterotopic quadruplet gestation with laparoscopic resection of ruptured interstitial pregnancy and subsequent successful outcome of triplets. Am J Obslet Gynecol. 1995; $172: 216$.

31. Sohail S. Haemorrhagic corpus luteum mimicking heterotopic pregnancy. J Coll Physicians Surg Pak. 2005;15:180-1.

32. Tamai K, Koyama T, Togashi K. MR features of ectopic pregnancy. Europ Radiol. 2007;17(12):3236.

Cite this article as: Bhattacharya R, Raut R, Kumari $\mathrm{B}$, Hojai DC. A rare case of heterotopic pregnancy: an avalanche of catastrophe. Int J Reprod Contracept Obstet Gynecol 2020;9:4702-6. 